\title{
La société française à travers
}

la chanson

\author{
Virginia Boza Araya ${ }^{1}$ \\ Universidad Nacional, Costa Rica
}

\section{RESUMEN}

La canción es una expresión popular ligada a las culturas y a las tradiciones del pueblo. Suele acompañar la evolución de la sociedad evocando sus alegrías, tristezas, decepciones y demás hechos de su vida cotidiana. En el campo de la didáctica del Francés como lengua extranjera, la canción of rece a los profesores la posibilidad de diversificar y ampliar las perspectivas y las aplicaciones sociolingüísticas en la clase. Un análisis minucioso realizado en dos mil canciones entre 1970 y 2003 permitió estudiar la evolución de la sociedad francesa. Los resultados de este estudio recopilados en La chanson, miroir d'une société demuestran los ef ectos del desarrollo económico en la sociedad.

\section{RÉSUMÉ}

La chanson a toujours été une expression profondément populaire, ancrée dans les cultures et les traditions des peuples. Elle suit l'évolution de la société et des individus en évoquant autant leurs joies, leurs malaises que tous les problèmes qu'ils affrontent au quotidien. Dans le domaine de la didactique du Français langue étrangère, la chanson offre aux enseignants la possibilité de diversifier, d'élargir les approches et les applications sociolinguistiques dans la classe. Une analyse approfondie effectuée sur 2000 chansons des années 1970 à 2003 a permis de suivre l'évolution de la société française. Les résultats de cette étude rapportés dans La chanson, miroir d'une société soulèvent les effets du développement économique sur la société.

\footnotetext{
I Correo electrónico: dantrou@hotmail.com.
} 
Palabras clave: canción popular, cultura, Francés como lengua extranjera, sociedad francesa

Mots-clés : chanson, culture, Français langue étrangère, société française

La chanson a toujours été une expression profondément populaire, ancrée dans les cultures et les traditions des peuples. La chanson, en tant qu'expression orale, est synonyme de mémoire, d'histoire des hommes. Elle représente surtout l'expression des sentiments les plus profonds de l'être humain. Indissociable donc de la société, la chanson est devenue aussi un outil pédagogique incontournable.

Dans le domaine de la didactique du FLE, la chanson offre aux enseignants la possibilité de diversifier, d'élargir les approches et les applications sociolinguistiques dans la classe FLE. Son utilisation ne peut que favoriser l'apprentissage de la langue et de la civilisation tout en motivant les apprenants.

Dans cet article, la chanson est analysée sous un volet sociologique afin de découvrir les aspects civilisationnels qui pourraient être exploités dans le but de renforcer les connaissances linguistiques et de faire acquérir des compétences culturelles aux futurs enseignants de français.

Lorsqu'on veut aborder la société française en classe FLE, la chanson s'avère, non seulement, une source inépuisable d'informations mais aussi un outil pédagogique très utile pour la faire découvrir. En fait, selon Yves Duteil la chanson est indissociable de la vie des personnes car elle les suit depuis la naissance jusqu'à la mort. « Les chansons, c'est nous, c'est notre histoire. Ça nous raconte " ${ }^{2}$. Elle suit l'évolution de la société et des individus en évoquant autant leurs joies, leurs malaises que tous les problèmes qu'ils affrontent au quotidien.

Yves Duteil, « Evolution de la langue française - Langue française et chanson » in : Jean-Marc Léger. Jean-Louis Roy. Dominique Perben, Michel Alliot, Tom Amadou Seck, Benjamin Dessus. Jacques Maurais, L'année francophone internationale (Québec : L'année francophone internationale. 1995) 51-53. 
Angèle Güller affirme que la chanson permet de « suivre les moindres remous de l'époque et les réactions des groupes et des individus aux événements » ${ }^{3}$. L'étude La chanson, miroir d'une société ${ }^{4}$ réalisée en 2003 soulève les effets néfastes du développement économique sur la société. Détérioration physique de la ville, crise des valeurs morales et crise économique marquent cette évolution.

\section{Une certaine image de la France}

La perception de la société française évoquée dans plusieurs chansons n'est pas très positive. Les chanteurs sont assez critiques quant à l'évolution de celle-ci. A la détérioration physique de la ville s'ajoutent des changements dans le comportement des citadins qui sont devenus indifférents, lassés et méfiants. Le chanteur Francis Cabrel le remarque dans la chanson $M a$ ville ${ }^{5}$ (1977). Ces attitudes sont également dénoncées par Louis Chédid dans les années 97 dans la chanson $I c i$ :

Ici, nez à nez, face à face dans le métro,

Ici, collés, serrés, presque peau contre peau

Du bruit,

Mais pas un sourire, pas un $\operatorname{mot}^{6}$.

Constatation qui fait transparaître un certain désespoir «Qu'ici, c'est pas une vie ». Ce phénomène ne fait que s'accentuer dans les

$3 \quad$ Angèle Güller, "Communication » in : Guy Calvet, Louis-Jean Bontempelli, Jacques Robin, Math Samba, Jeanne Tony, Anne Zumkir, Colloque international sur la chanson de langue française (Bruxelles: Commission française de la Culture de l'Agglomération de Bruxelles, 1979) 26-28.

4 Virginia Boza Araya, La chanson, miroir d'une société. Cette étude présente les résultats d'une analyse approfondie effectuée sur 2000 chansons des années 1970 à 2003 dont le but était de suivre l'évolution de la société française. L'image de la société française de cette période n'est pas très positive (2003).

Francis Cabrel, Ma ville (1977).

Louis Chedid, Ici (1997). 
années 80/90. L'évolution de ces sentiments laisse présager une crise des valeurs morales et aussi la crise économique des années 80 . Dégradation qui est bien expliquée par Pierre Perret dans $Y$ a pas de malaise (1983). Le malaise règne dans tous les domaines de l'activité économique : au niveau commercial les produits comme le camembert se vendent moins bien. Le niveau de vie, aussi bien des entrepreneurs que des cadres, n'est plus aussi réjouissant qu' on pourrait le penser. Le déficit s'accentue car certaines industries fonctionnent moins bien. Pour combler ce déficit certains réclament «Il faut trouver des milliards » (Les années 30, Michel Sardou - 1982). L'État a recours à des dévaluations pour empêcher l'inflation et pourtant « la vie augmente » clame Michel Sardou (Les années 30-1982). Selon Pierre Perret une autre conséquence de la crise est l'affaiblissement du franc par rapport au dollar. Les impôts continuent d'enfoncer les Français et même les $\mathrm{PDG}^{7}$ d'entreprises.

\section{Temoins privilegies de la crise : les chanteurs}

Les chanteurs subissent aussi la crise. Dans leurs chansons, ils laissent transparaître leur mécontentement. Certains dénoncent les prélèvements excessifs du Ministère des Finances spécialement à l'égard des chanteurs. Il n' est donc pas étonnant que dans Cow boy, Jean Leloup compare le Ministère des impôts à des hommes d' affaires immoraux qui essaient de tout lui prendre même sa guitare. Les Inconnus (Rap tout), passent en revue tous les impôts et taxes diverses que le contribuable français doit payer. Il faut travailler pour payer les impôts car une partie non négligeable du revenu part dans les caisses de l'Etat. Cette imposition provoque une grande frustration qui pousse de plus en plus de personnes, d'entrepreneurs et de chanteurs à vouloir aller vivre ailleurs. En fait, les démêlés des chanteurs tels qu'Alain Barrière, Michel Polnareff et plus récemment, Florent Pagny illustrent

Présidents Directeurs Généraux. 
ce phénomène. Dans sa chanson Ma liberté de penser (2002) Pagny dénonce l'emprise de l'Etat qui a des conséquences tragiques. Le texte de Pierre Perret est très intéressant du point de vu socio-linguistique car il montre l'image de la France en crise, évocation qui est loin d'être celle présentée dans des textes plus idylliques. Eddy Mitchell, lui, est très ironique lorsqu'il évoque ses rapports avec le Fisc. Il leur consacre deux chansons : Lèche-bottes blues et Ne changeons rien :

J'suis escroqué comme un touriste

Ma feuille d'impôt est un jeu d' piste...

$\mathrm{Ne}$ changeons rien!

On vit une époque fantastique

Le groupe ATK est encore plus âpre dans son jugement du fisc car il considère que l'Etat « rackette » les citoyens y compris les chanteurs.

Cette vision d'une société aux valeurs inversées est également dénoncée par le groupe Fonky Family (Sans rémission - 1997). Ils sont outrés de voir qu'il y a beaucoup de personnes qui s'épuisent au travail pour que l'Etat, les taxes et que les politiciens s'enrichissent avec le fruit de tant de sacrifices. D'ailleurs, ils sont assez sévères dans leur jugement car ils considèrent que «L'État est de loin le plus grand proxénète ».

\section{Bouleversements sociaux}

Chanteurs piégés, société en proie au pessimisme et aux conséquences qui s'en suivent. Cette crise va plonger les Français dans le désarroi ${ }^{8}$, celui de la jeunesse des banlieues, constaté par le chanteur

\footnotetext{
$8 \quad$ Suprême NTM, Qu'est-ce qu'on attend? (1995) : MC Solaar, Quand le soleil devient froid (1997) : Arsenik, La rue t'obsenve (1997) ; Fonky Family, Cherche à comprendre (1998) ; Arsenik, Ma vision du monde et Une saison blanche et sèche (1998) ; Cercle Rourge, La main tendue (2000).
} 
Passis et que le groupe Suprême NTM remarque dans la société française dans sa chanson Qu'est-ce qu'on attend? (1995):

Voilà pourquoi cela finira dans le désarroi Désarroi déjà roi, le monde rural en est l'exemple Désarroi déjà roi, vous subirez la même pente L'agonie lente

D'autres problèmes sont soulevés par les chanteurs. Pierre Perret, par exemple, évoque les problèmes de natalité dû au fait que les femmes ont moins d'enf ants et du vieillissement de la population entre autres. Déjà en 1978 Michel Sardou exprimait cette inquiétude dans la chanson 6 milliards 900 millions 980 mille car il constatait que la natalité restait déficitaire à cette époque et constituait une menace latente. La France deviendrait-elle un peuple en voie d' extinction dans l'avenir.
Mais j'aimerais que quelqu'un vienne m'expliquer pourquoi, Nous, les champions de l'amour, Nous en resterons toujours A n'avoir seulement que 50 millions de Gaulois.

A cet égard Pierre Perret se fait l'écho de Michel Sardou mais, contrairement à ce dernier, il explique les causes de ce changement de mentalité dans la société. Il l'attribue, en particulier, à une évolution de la mentalité féminine qui devient plus libérale « toutes les minettes prennent la pilule ». Ainsi le déclarent certains chanteurs ${ }^{9}$ tels que

\footnotetext{
') Patrick Juvet, Où sont les femmes ? (1977) ; Charles Aznavour, Inoubliable (1994); Thomas Fersen, Bijo, (1980) ; Jean-Pierre Ferland, Les courtisanes (1980) : Gérard Depardieu, Où sont le's femmes de capitaine (1980) ; Dalida, Femme (1983) ; Mylène Farmer, Xxl (1986) : Alain Chamfort, J'entends tout ; Charlélie Couture, Rachel (Elle adore Pandore): Juliene Clerc,
} 
Cooky Dingler qui fait le portrait des femmes modernes dans sa chanson Femme libérée. Ce sont des femmes insouciantes et frivoles qui ont des aventures passagères, qui ne s'inquiètent que de leur apparence physique et qui croient tout savoir :

Au fond de son lit un macho s'endort

Qui ne l'aimera pas plus loin que l'aurore

Mais elle s'en fout, elle s'éclate quand même

Il rejoint, sur ce point, l'opinion sur les Parisiennes qu'avait déjà, dans les années 1976, Marie-Paule Belle (La Parisienne $\left.{ }^{10}\right)$. Par ailleurs, d'autres chanteuses comme Mylène Farmer (Libertine 1986) a vouent, sans complexe, qu'elles tiennent à leur liberté sexuelle :

$\mathrm{Je}$, je, suis libertine

Je suis une catin

Aussi, cette natalité déficitaire, laisse-t-elle prévoir à long terme les problèmes des retraites dont la France subit les conséquences à présent. Il suffit de suivre l'actualité : les grèves du premier semestre 2003 montrent que le problème des retraites est loin d'être résolu. Par ailleurs, le spectre du chômage, des jeunes en particulier, rôde et va se confirmer dans les années 90 et 2000. Michel Sardou faisait déjà allusion en 82 (Les années 30) à la loi des 35 heures de travail : des conflits entre les entreprises et le gouvernement se multiplient, bien que certains y trouvent leur compte. Ce sujet sera repris et exploité par Sniper en 2003 dans sa chanson 35 heures. La chanson devient aussi une sorte de chronique des faits marquants de l'actualité, de cette évolution rapide de la société prise dans le marasme de la globalisation.

Hélène ; Gilbert Bécaud, Désirée (1982); Alain Bashung, Madame rêve ; Pierre Bachelet, Les Lola (1992).

Marie-Paule Belle (musique) ; F. Mallet-Joris, Michel Grisolis (paroles) (1976) ; La Parisienne ; Allo Music. 
A partir des années 80 la morosité s' installe en France et d'autres problèmes sociaux apparaissent. C' est ce que soulèvent les chansons analysées. Par exemple, le problème de la pauvreté, de plus en plus évident dans les banlieues et dans la rue est mis sur le tapis. Les familles vivant dans les cités de $\mathrm{HLM}^{11}$ sont plus que jamais défavorisées ; la plupart ne subsistent que grâce aux allocations de chômage versées parl'État ou le RMI (Revenu minimum d' insertion) ${ }^{12}$, et se caractérise par une cellule familiale monoparentale. La multiplication des collèges et lycées classés ZEP (zone d'éducation prioritaire) en est la preuve. Bon nombre de chanteurs se sont trouvés, lorsqu'ils étaient petits, seuls avec leurs mères et leurs frères. C'est le cas de MC Solaar (Lève-toi et rap-2001) qui, tout comme le chanteur du groupe Fonky Family (Maintenant ou jamais - 1998) décrivent les cauchemars de leur vie démunie, les difficultés subies par leurs mères qui ont dû travailler durement pour élever leurs enfants.

Un autre exemple parmi d' autres est rapporté par le groupe IAM dans la chanson Femme seule (1993). C'est le parcours difficile de beaucoup de jeunes filles de la banlieue, qui de surcroît, sont des filles d'immigrés. Issues d'une famille nombreuse, elles n'ont pas pu poursuivre des études, la rue devenant leur unique école. Elles se marient souvent très jeunes. Les enfants arrivent vite et les maris les abandonnent au bout des quelques années de mariage. Elles restent donc seules avec leurs enfants et doivent assumer leur charge. Ce qui explique le nombre croissant des familles monoparentales en France, dont le chef de famille est dans la majorité des cas une femme. Elles assument leur rôle, tant bien que mal, avec beaucoup de dignité et à force de nombreux sacrifices. On remarque chez les chanteurs un sentiment de tendresse et d'admiration envers ces jeunes femmes.

La pauvreté frappante dans la rue ne laisse pas indifférents les chanteurs comme Renaud ${ }^{13}$ qui dans sa chanson Le gringalet

$11 \quad$ Habitation à loyer modéré.

: Chanson plus bifluore (1994): Chômage au fond de la vallée: Akhénaton, Lettre aux hirondelles (1996) : Renaud, Le parfait citoven (1997) : Tryo. Salut ô (2002).

13 Arsenik, Chñsanthèmes (1998); ATK, Paraîre (2000) : Fred Alpi, Ici et Maintenant (2000). 
$(1985)^{14}$ se penche sur le cas des indigents qui meurent de faim et de froid. Cette déchéance humaine est également dépeinte par le chanteur MC Solaar (Armand est mort - 1991) qui dénonce les malheurs de certaines personnes qui, pour des multiples raisons, et en particulier parce qu'elles se retrouvent au chômage, sont rejetées par leurs familles. Ne pouvant plus assumer les dépenses, elles grossissent les rangs de gens qui vivent dans la rue. Notons à cet égard que la solidarité dans le couple n' est pas de mise. Ces personnes en grande difficulté sociale sont souvent confrontées à toutes sortes de violence urbaine et de misère humaine au point d'en mourir. D'autre part, les valeurs morales ne sont plus de rigueur. Le matérialisme globalisant s'impose dans l'esprit des individus en général et des jeunes en particulier ${ }^{15}$. Selon Michel Sardou (Atmosphères - 1984) ils éprouvent une sorte de désenchantement qui les pousse à voler, à se comporter comme des voyous, à prendre la vie à la légère pour tromper leur désespoir car ils n'en voient pas d'issue. L'ombre du chômage plane toujours. C'est pour cette raison que beaucoup de jeunes ne souhaitent pas faire des études car ils n'ont aucune perspective d'avenir.

Par conséquent, ils rêvent de pouvoir partir ailleurs. Cette déchéance des jeunes est dénoncée sans ambiguïté, dans les années 90 , par Daniel Balavoine dans Petite Angèle. Il s' interroge sur la violence de la jeunesse des cités qui casse tout. Il ne comprend pas sa réaction.

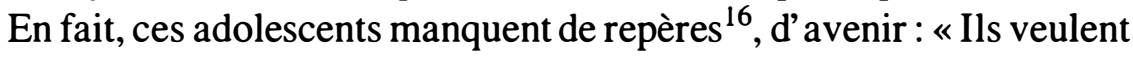
savoir vers quoi on les entraîne ". La chanteuse Assia (Laissez-nous rêver - 2000) (comme d'autres artistes ${ }^{17}$ ) confirme ce postulat de Daniel Balavoine en les caractérisant de :

\footnotetext{
14 ATK, Paraître (2000) : Chanson plus Bifluoré, Chômage au fond de la vallée (1994); Cercle Rouge, La main tendue (2000).

15 Assassin, Note mon nom sur ta liste (1991) : Suprême NTM, L'argent pourrit les gens (1991). Assassin, La flamme s'éteint (1995).

16 Assassin, L'histoire suit son cours (1991) : Suprême NTM. Qu'est-ce qu'on attend?

17 Suprême NTM, Qu'est-ce qu'on attend? (1995): ATK. Les Histoires se passent (2000).
} 
Génération désorientée,

Sur l'échelle des valeurs inversées

\section{Un theme recurrent : la violence}

La détresse évoquée précédemment s'exprime aussi par la violence présente dans toutes leurs actions, ce que Renaud affirme dans La Ballade de Willy Brouillard en 1984. Cette chanson évoque la galère vécue dans les banlieues autant par les policiers que par les jeunes. En effet, les tensions et la brutalité des actions mettent en danger celui qui s'y aventure. C'est pourquoi le chanteur la définit comme « une jungle à la con ». Le groupe Cercle Rouge en a une vision plus noire dans sa chanson Mavision dumonde (2000). Il affirme avec véhémence que «mon paradis n'est qu'un enfer de béton sur terre ».

Un autre portrait de la jeunesse des cités est brossé par MC Solaar dans L'argent ne fait pas le bonheur en (1998). Selon lui, les jeunes veulent le luxe, manger du foie gras, frimer avec de belles voitures, les affaires faciles, pouvoir imiter les riches sans que cela ne leur coûte rien. Cette vision de la jeunesse paumée est partagée par le groupe Fonky Family. Il proclame dans sa chanson Sans rémission (1997).

Je veux faire du fric

Rien qu'en respirant

Et manger mieux que bien...

On a envie de se faire de millions

Sans se poser des questions...

L'envie de vivre dans le luxe

Tous les chemins sont bons pour réussir

Il semblerait que les jeunes des années 90 sont obsédés par cette idée de devenir riche coûte que coûte mais sans pour autant se tuer à la tache comme l'ont fait leurs parents. Ce désir découle du fait qu'ils voient partout comment certaines personnes s'enrichissent grâce à des 
affaires illégales, à des trafics divers, ou tout simplement parce qu'ils ont hérité une grosse fortune. Aussi, confirment d'autres artistes, ce même sentiment est inspiré par l'injustice de la vie ${ }^{18}$.

Ces jeunes ne reconnaissent plus les liens familiaux ou amoureux, seul les amis comptent. Le groupe Manau en donne un exemple dans sa chanson La confession - (1998) : c' est l'histoire des jeunes des banlieues tués qui, dans leurs dernières minutes, font le bilan de leur vie. Leur seule attache restent leurs amis, semble-t-il. Ils sont souvent bouleversés par la mort de l'un d'entre eux. MC Solaar signale également la désintégration familiale. Le constat: un fossé s'est creusé entre ces adolescents et leurs familles. Les parents ne reconnaissent plus leurs enfants. Cette désagrégation ${ }^{19}$ est mise en évidence non seulement par MC Solaar en 1997 dans la chanson déjà citée ci dessus mais également par Assia (Laissez nous rêver - 2000) qui souligne :

Des mères en pleurs, effarées

Dans leur chair le Mal s'est immiscé

Se meurent de voir les anges tomber

Les jeunes se laissent facilement séduire par la délinquance depuis un très jeune âge. Cette faiblesse est très bien expliquée par le groupe IAM dans plusieurs chansons mais tout particulièrement dans Petit Frère. Il y trace le parcours suivi par des enfants de 10 ans qui, à cet âge, ont déjà appris l'intolérance. Ils pratiquent une violence gratuite dans le but d'imiter les adultes. Ils se complaisent à jouer les sauvages, au « Mortal Kombat » et ont déjà eu des relations sexuelles. A 13 ans ils deviennent des « Caïds » pour se procurer tout ce qu'ils

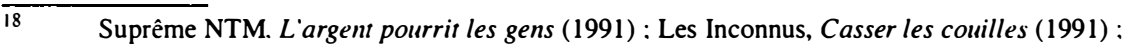
Akhénaton. Bad Boys de Marseille - Lettre aux hirondelles (1996); MC Solaar, Le sens de la vie (1997) : IAM. Nés sous la même étoile (1997): MC Solaar, L'argent ne fait pas le bonheur (1998) : IAM. Marseille la nuit (1999). 
désirent et qu'ils ne peuvent pas s'acheter. Le chanteur Sniper le confirme aussi, de même que MC Solaar dans sa chanson La France (2001).

La délinquance augmente

même les plus jeunes s'y mettent

Pètent des bus parlent de braquage

Et à l'école ils rackettent

Ce groupe, commebeaucoup d'autres, attribue cette dégringolade de l'enf ance à trois éléments importants de la société qui sont liés entre eux. Le premier découle de l'attitude des adultes qui, par négligence ou par égoïsme, ont " montré que faire le mal, c'est bien ». Cette responsabilité est partagée par les Média qui, à force de passer des images brutales, des reportages ou des films où le sang et la mort règnent en maîtres, banalisent ces faits regrettables. Et enfin, à la société de consommation dont la cible parfaite ce sont les enfants, marché qui rapporte à moyen et à long terme.

Nombreux sont les témoignages où violence et froideur entrent en jeu : rivalités de gangs, questions d'honneur, règlements de comptes, trafics en tout genre, etc. Ils tuent sans la moindre hésitation. Assia (Laissez nous rêver - 2000) le décrit dans ces termes :

Une question d'honneur et de fierté

C'est là que ce môme a tiré

Dans ses yeux, pas l'ombre d'un regret

Les causes de cette grave situation sont multiples. Tout d'abord, un environnement violent et étouffant ayant pour toile de fond du béton gris et froid. Le groupe Cercle Rouge s' est fait le porte-parole de ces jeunes pour dénoncer cette triste réalité dans sa chanson $M a$ vision du monde (2000) : " Je viens de coin du monde où on habite les uns sur les autres ». Cette grisaille est la source d'un sentiment de frustration profond car ils ne voient pas d'avenir pour eux : 
Autour de moi rien ne va plus, c'est cause perdue

Même ce qu'ils voient en dehors de la cité ne les rassure pas. C'est ce qu'explique MC Solaar dans Le sens de la vie (1997). Cette réalité peut également porter préjudice aux jeunes qui poursuivent des études ${ }^{20}$. Ils risquent de se trouver au chômage. Dans les banlieues, la pauvreté est partout, la plupart des familles gagnent le RMI ou sont demandeurs d'emploi, les dettes s'accumulent. Ils sont ainsi dépouillés du peu qu'ils possèdent par des huissiers qui viennent souvent saisir ce qu'ils peuvent. Freko, du groupe ATK, s'exclame avec un certain désespoir « Je suis une crève la faim ${ }^{21}$. Cette situation renforce ce profond sentiment d'injustice alors que certains vivent dans l'opulence ils souffrent. Cette richesse est vécue comme un affront qu' ils subissent constamment et qui les pousse à la délinquance. Est-ce aussi un appel au secours ? D'ailleurs Assia l'affirme (Laissez nous rêver - 2000).

Soyez donc fiers de vos bébés

C' est vous qui les avez engendrés

Dans cette société matérialiste où la valeur d'une personne ne se mesure que par ce qu'elle possède, ou comme le dit Louis Chédid (Ici - 1997) «Le sens des valeurs c'est le sens des affaires ", les jeunes désorientés se laissent aller au «biz ». Ces inégalités vont provoquer une série d'exactions où les plus faibles en seront les victimes. Les jeunes vont imiter leurs nouveaux héros, n'ont pas ceux qui luttent pour le bien mais ceux qui possèdent l'argent et le pouvoir : de grands trafiquants de drogue comme Pablo Escobar, des stars de films pornographiques... D'ailleurs, il n'y a pas que les malfaiteurs

Cercle Rouge, La main tendue (2000); ATK, Vingt ans (2000).

21 MC Solaar, A temps partiel (1991); Akhénaton, Bad Bơıs de Marseille (1995); Akhénalton, Lettre aux hirondelles (1996); KDD, Une couleur de plus au drapeau (1998); Akhénaton. Lettre aux hirondelles (1996). 
connus qui agissent de la sorte, des hauts fonctionnaires sont aussi mêlés à des affaires illégales. Renaud le dénonce déjà dans La Ballade de Willy Brouillard (1994) :

Y protège l'État, les patrons

Ceux qui refourguent la came aux fistons

Il semblerait que la corruption est partout. Trois mots dans cette énumération suffisent à MC Solaar pour insister sur cette corruption :

Scandale égal commercial

Dopage à tous les niveaux sportifs

Ou des courses truquées

Dans les textes des années 90 - 2000 la violence est de plus en plus présente. Que ce soit dans les actions ou dans les paroles, la déchéance de la société s'aggrave. Les jeunes et même les enfants deviennent de plus en plus agressifs, imposant l'enfer dans leur banlieue. Ainsi le proclame le chanteur Akhénaton dans sa chanson Lettre aux hirondelles (1994).

Les mômes n'ont que 16 ans et imposent la terreur dans les blocs... Être un âne à l'école n'est plus à la mode Par contre plus qu'avant, être une racaille Une canaille

Certains essaient de s'en sortir. Ils sont conscients qu'ils sont victimes des stéréotypes qui leur donnent l'image de délinquants, de fainéants. Il faut savoir aussi sur que la plupart de ces jeunes de milieux défavorisés sont des fils d'immigrés. Le groupe IAM explique :

J'traîne la reput' de Mars sur mes épaules

Ces gosses qui fréquentent la rue 
Ces gosses qui fréquentent l'école

Et ceux qui jouent les killers ${ }^{22}$

En fait, plusieurs facteurs engendrent cette violence chez les jeunes. Tout d'abord le sentiment d'injustice face aux inégalités que la société, elle-même, cautionne et qui les exclut ; en particulier ceux qui sont issus de familles d'immigrés. Ce racisme ${ }^{23}$ est flagrant dans l'attitude des policiers qui se manifeste en tout premier lieu par un contrôle systématique de leur identité :

Vos papiers, contrôle d'identité-

Formule devenue classique à laquelle

Tu dois t'habituer

Seulement dans les quartiers

Les condés de l'abus de pouvoir ont abusé

Les chansons dénoncent cette attitude et les sentiments que cet abus a fait ressortir chez ces jeunes français. Certains chanteurs évoquent ces exactions dans leurs chansons. Ce thème de la répression implacable devient un leitmotiv dans leurs chansons ${ }^{24}$. Cette violence policière n'est pas sans conséquences : bavures et même des jeunes morts, dans certains cas, ne sont pas rares d'après les chanteurs. L'un des cas le plus médiatisé est celui de Malek Oussekine, que le groupe Assassin rappelle dans sa chanson L'État assassine.

\footnotetext{
I2 Assassin, Peurd'une race (1993), IAM. Marseille la muit. 1999 : ATK. Paraître, 2000 ; Sniper. Pris pour cible, 2001. Saian Supa Crew. Toumer la page (2001).

23 Assassin (1991), Esclave de votre société : Assassin, Peur d'une race 1993, L'État assassine' 1995.

Renaud.Fallait pas,1995 ; Arsenik. Éclater un type des Assedics - Partout la même, 1997 : 1995 ; Suprême NTM, Police, 1995 : KDD. Qui sera le prochain ?, 1998. KDD. Aspect susprect'. 1998 ; Assassin. État policier, 2001, Saïan Supa Crew. Police, 2001 : Lunatic. Mcun'ais tran'ail. 2001 ; Pierpoljak. Police, 2002.
} 


\section{L'État assassine, un exemple}

Malek Oussekine

Bin, bang_, la police est comme un gang

Car l'État assassine Makomé

En a été la victime

Ce cas est également mentionné par le groupe Saïan Supa Crew l'évoque dans sa chanson Tourner la page (2001). Il n'est pas le seul à stigmatiser l'action de la police puisque d'autres jeunes ont subi le même sort. Dans la chanson État policier (2000), le groupe Assassin clame pour que ces bavures policières cessent, car il y a trop de victimes.

L'état policier n'a pas épargné

la vie de Bouziane Abdel Kader

$\mathrm{Ni}$ celle de Jawad Zaouiya, paix à leur famille

Police au nom de tous les petits voleurs

Que tu as tués en rigolant...

Y aura Farid y aura Djamel imagine la fanfare

D'ailleurs, certaines de ces bavures ont provoqué des émeutes, des vitres cassées, des voitures incendiées, des blessés lors d'affrontements entre la police et les jeunes excédés. Ces morts, ces manifestations violentes, ces contrôles injustifiés laissent les banlieues meurtries déchaînant un sentiment de Haine ${ }^{25}$, sentiment qui ne s'estompe pas au cours de leur existence. La société française devient une sorte de poudrière susceptible d'éclater à tout moment puisque même dans les chansons les jeunes expriment une sorte de menace.

$\approx \quad$ Assassin (1995). La flamme s'éteint ; NTM. Police, 1995 : Assassin, L'Etat assassine 1995 : Kent. La haine est là, 1996 : Arsenik. Jour 2 tonnerre, 1997 : Akhénaton. Un brin de haine, 1996 : IAM. Marseilles la nuit, 1998 : Arsenik. Ils m'appellent. 1998 :Pierpoljak. Police, 2000 : ATK, Paraître, 2000 : ATK, Haine, 2000 Saïan Supa Crew. Toumer la page, 2001. 
Cette explosion annoncée devient réalité au cours du $1^{\text {er }}$ semestre $2005^{26}$. Les chanteurs ne se trompaient pas, ces jeunes désœuvrés avaient plongé la France dans une sorte d'état de siège. Le chanteur Damien Saez exprime, en 2002, une sorte de menace :

Moi j'veux du nucléaire

Je veux du sexe et du sang

Des bombes dans le RER

Même si je ne suis qu'un enfant

L'État qui, cautionne les actions de ces policiers, est également pris pour cible par des rappeurs qui le perçoivent comme un appareil d'oppression qui ôte leur liberté d'expression. La censure ${ }^{27}$ de leur texte est permanente au point que certains groupes se sont vus condamner pour incitation à la violence et leurs chansons interdites de diffusion. Censure que Akhénaton dans son texte Dangereux. Il déplore en effet que les groupes de rap soient poursuivis en justice à cause de leurs textes jugés réactionnaires. La violence de leurs mots attise la haine contre les policiers et l'État. Il en va de même pour des institutions publiques comme les ASSEDICS ${ }^{28}$ qui font preuve de racisme, semble-t-il, envers certaines personnes affichant un certain mépris pour elles en raison de leur origine. Ils bloquent bien souvent leur dossier sciemment.

Les droits fondamentaux de l'homme ne sont plus respectés par l'État français qui met en avant l'égalité et la fratemité sans en

26 CF. Gas. Valérie (2005). Banlieues: des violences «organisées». RFI ; Émeutes dans les banlieues françaises : Face aul désespoir, seulc la lutte de classe est porteuse d'avenir ; Tubiana, Michel (2005). Etat d'urgence : qui sont les ennemis ? Le Monde. Peyrat. Didier (2005). Incendiaires et cogneurs : Glucksmann, André (2005). Les feux de la haine. Le Monde : Gas, Valérie (2005). Le couvre-feu face à la violence. RFI. Leclerc, Jean-Marc (2006) Les émeutes dans les banlieues ont fait flamber les statistiques. RFI.

26

27 Assassin. Kique ta merde (1993) ; IAM, Dangereux (1997) ; Suprême NTM, On est encore là (1998) : Assassin, État policier (2000).

ASSEDIC, Association pour l'emploi dans l'industrie et lë commerce. Organisme paritaire patronat-syndicats, créé par une convention collective (déc. 1958), qui assure aux travailleurs sans emploi en France une indemnisation complémentaire de l'aide publique. 
donner l' exemple. Ainsi, censure et racisme vont de pair selon certains chanteurs de rap, c'est le cas du groupe NTM qui le dit clairement :

On nous censure parce que

notre culture est trop basanée

Qu'on ne représente pas assez la France

D'une manière générale les chanteurs de rap deviennent des défenseurs de gens en difficulté qui vivent dans les cités défavorisées qui sont souvent des laissés pour compte de l'État. Cet appel à la justice sociale rappelle l'engagement d'autres chanteurs des années $70 / 80$. Renaud invoquait « le peuple comme figure à la fois sociologique (qu'il faut décrire) et politique (qu'il faut célébrer) » et qu'il faut défendre. C'est la préoccupation principale des rappeurs qui veulent mettre en évidence cette réalité difficile que vivent ces jeunes. Ils soulignent leurs qualités et leurs souffrances. Le rap est toujours apprécié par les jeunes et les moins jeunes par son caractère actuel, pour sa quête de justice, pour sa constante dénonciation de l'exploitation économique et sociale l'aliénation culturelle. La différence avec les années 70 c'est que le peuple, d'une certaine manière, prend les armes autrement.

En fait, ces chanteurs cherchent surtout à surmonter les difficultés à travers la musique et plus particulièrement à travers les paroles, sorte de planche de salut. La langue française représente pour eux une arme pour combattre la corruption qui gangrène la société et qui la plonge dans le chaos. C'est pourquoi, pour certains groupes, la langue devient l'outil par excellence. Le travail linguistique et stylistique est à la base de leurs compositions. La langue prend une place plus importante que la musique. Dans un article, publié le 18 septembre 2003 dans Libération, Akhénaton du groupe IAM considérait que les rappeurs actuels se doivent de « redonner ses lettres de noblesse au rap faire sonner les mots. Il insiste sur le fait que le rap est « une musique ultra mobile » comme l'est le français qui est en constante mutation.

170 